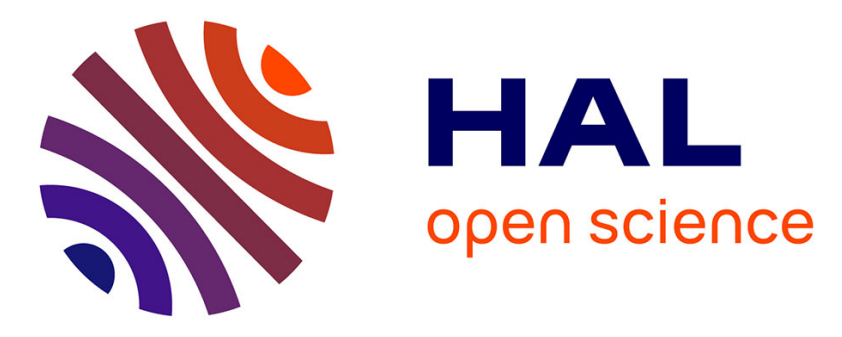

\title{
Transition from Saltation to Collisional Regime in Windblown Sand
}

Velotiana Jean-Luc Ralaiarisoa, Jean-Baptiste Besnard, B. Furieri, P. Dupont, Ahmed Ould El Moctar, F. Naaim-Bouvet, Alexandre Valance

\section{- To cite this version:}

Velotiana Jean-Luc Ralaiarisoa, Jean-Baptiste Besnard, B. Furieri, P. Dupont, Ahmed Ould El Moctar, et al.. Transition from Saltation to Collisional Regime in Windblown Sand. Physical Review Letters, 2020, 124 (19), pp.198501. 10.1103/PhysRevLett.124.198501 . hal-02796938

\section{HAL Id: hal-02796938 \\ https://hal.science/hal-02796938}

Submitted on 18 Jun 2020

HAL is a multi-disciplinary open access archive for the deposit and dissemination of scientific research documents, whether they are published or not. The documents may come from teaching and research institutions in France or abroad, or from public or private research centers.
L'archive ouverte pluridisciplinaire HAL, est destinée au dépôt et à la diffusion de documents scientifiques de niveau recherche, publiés ou non, émanant des établissements d'enseignement et de recherche français ou étrangers, des laboratoires publics ou privés. 


\title{
Transition from saltation to collisional regime in wind-blown sand
}

\author{
J-L. Ralaiarisoa ${ }^{1,5}$, J-B. Besnard ${ }^{1,2}$, B. Furieri ${ }^{3}$, P. Dupont ${ }^{2}$, A. Ould El Moctar ${ }^{4}$, F. Naaim-Bouvet ${ }^{5}$, A. Valance ${ }^{1}$ \\ 1 Univ Rennes, CNRS, Institut de Physique de Rennes, UMR 6251, 3500 Rennes, France \\ 2 Univ Rennes, INSA Rennes, LGCGM, 3500 Rennes, France \\ 3 Universidade Federal do Espírito Santo, DEA, 29060-970 Vitória, ES, Brazil \\ 4 Univ Nantes, Laboratoire Thermique et Energie, UMR 6607, 44306 Nantes Cedex, France \\ 5 Univ. Grenoble Alpes, INRAE, UR ETNA, 38000 Grenoble, France
}

(Dated: April 20, 2020)

\begin{abstract}
We report experiments on wind-blown sand that highlight a transition from saltation to collisional regime above a critical dimensionless mass flux or Shields number. The transition is first seen through the mass flow rate $Q$ which deviates from a linear trend with the Shields number and seems to follow a quadratic law. Other physical evidences confirm the change of the transport properties. In particular, the particle velocity and the height of the transport layer increases with increasing Shields number in the collisional regime while the latter are invariant with the wind strength in the saltation regime. Discrete numerical simulations support the experimental findings and ascertain that mid-air collisions are responsible for the change of transport regime.
\end{abstract}

Introduction - The study of wind-blown sand started seventy years ago with the seminal work of Bagnold [1]. He was the first to identify the dominant mode of sand transport, termed saltation, which consists of grains traveling in a sequence of ballistic trajectories and producing a splash of new ejected grains when colliding back onto the soil. The saltating particles do not undergo any mid-air collision. This is the peculiarity of the saltation regime of transport which is now well documented either experimentally or theoretically [2-4].

Saltation regime is expected to break down at high wind speed when the particle concentration within the transport layer is so high such that mid-air collision are likely to occur. Several recent numerical studies $[5,6]$ revealed indeed that at high fluid shear stress a highly collisional regime develops close to the bed surface. This collisional layer was referred to as a "soft" bed in [5]. Duran et al. [2] also identified through numerical simulations a change of transport regime at high wind speed. In the present letter, we provide for the first time experimental evidences that wind blown-sand undergoes a transition from the saltation regime to a collisional regime at high Shields number. We also developed discrete numerical simulations that give credit that the transition is driven by mid-air collisions.

Wind tunnel experiments - The experiments reported here were performed in a $6 \mathrm{~m}$ long wind tunnel with a cross-section of dimensions $0.27 m \times 0.27 m$ [7]. The floor of the tunnel is covered with a $2.5 \mathrm{~cm}$ thick layer of sand composed of quartz grains with a median diameter $d=190 \mu \mathrm{m}$ and a density $\rho_{p}=2650 \mathrm{~kg} / \mathrm{m}^{3}$. The nominal air velocity $U_{\infty}$ (i.e., the air speed outside from the boundary layer) in the wind tunnel was varied between 5 and $20 \mathrm{~m} / \mathrm{s}$, corresponding to Shields number $S^{*}$ ranging from 0.03 to 1.5. A typical experimental run lasts several minutes at a constant nominal velocity $U_{\infty}$ during which air and grain velocity profiles were measured by Pitot tubes and Particle Imaging Velocimetry $[7,8]$. Particle flux density profiles were also assessed via a particle counter probe (SPC-S7, Niigata Electric [9, 10]). At the end of each run, the sand collected at the exit of the tunnel was weighed and the resulting outgoing transport rate $Q$ estimated [11].

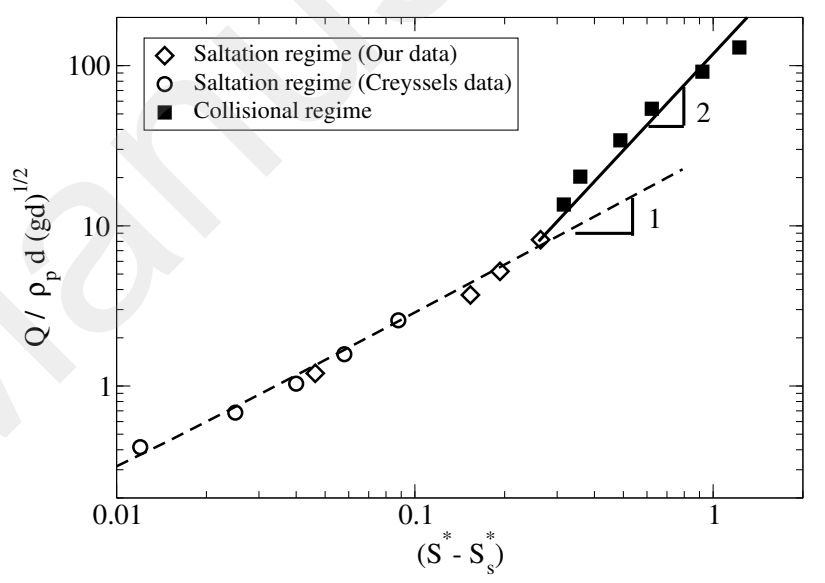

Figure 1: Dimensionless mass flow rate versus the

Shields number. The Shields number $S^{*}$ is a dimensionless number that compares the turbulent shear stress to the grain weight: $S^{*}=\rho_{a i r} u^{* 2} /\left(\rho_{p} g d\right)$ where $u^{*}$ is the friction velocity (extracted from the air profiles as explained in the caption of Fig. 2). Data by Creyssels et al. [8] obtained in longer wind-tunnel and corresponding to the saltation regime have been added to the graph.

The transition from saltation to collisional regime is clearly evidenced through the variation of the mass flow rate as a function of the Shields number $S^{*}$ (see Figure 1). Fig 1 shows clearly a change of behavior at a critical Shields number $S_{c}^{*} \approx 0.3$ (corresponding to a free stream velocity $U_{\infty} \approx 10.4 \mathrm{~m} / \mathrm{s}$ ). For $S^{*}<S_{c}^{*}$, the mass flow rate $Q$ scales linearly with $\left(S^{*}-S_{s}^{*}\right)$ (where $S_{s}^{*}=0.008$ is the Shields number at which transport ceases), whereas for $S^{*}>S_{c}^{*}$, it strongly deviates from a linear regime. In the latter regime, the variation of the flow rate is clearly a non-linear function of the Shields number: $Q \propto S^{* n}$ 
with $n>1$. The best fit to the data gives $n=1.8$ which is close to 2. Importantly, transport regime at very high Shields number (i.e., $S^{*}>0.7$ corresponding to Rouse number below 2 [14]) should be interpreted with caution since turbulence velocity fluctuations start to become important and particles may enter in suspension.

Another evidence of the regime change is seen in the vertical air velocity profile (see Fig. 2). The vertical air velocity profile $U(z)$ above the transport layer is well described by a logarithmic law as shown by Bagnold [1]: $U(z)=U_{f}+\frac{u_{*}}{\kappa} \ln \left(z / z_{f}\right)$, where $z_{f}$ and $U_{f}$ are respectively the height and velocity of the focus point defined as the locus where the air speed is independent of the wind strength, and $\kappa=0.41$ is the Von Karman's constant. This focus height $z_{f}$ can be interpreted as the height of the transport layer which is found to be independent of the wind strength [7] in the saltation regime. Our measurements confirm the existence of a focus point as long as $S^{*}<S_{c}^{*}$. We find: $z_{f}=40 \mathrm{~d}=8 \mathrm{~mm}$ and $U_{f}=3 \mathrm{~m} / \mathrm{s}$. However, above the critical Shields number

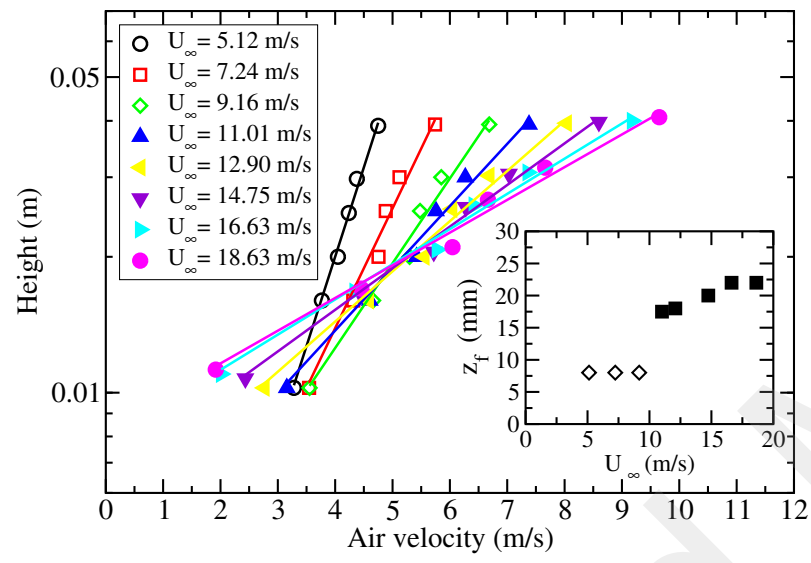

Figure 2: Air velocity profiles for different flow strengths: They exhibit a logarithmic increase and are well approximated by $U(z)=(u * / \kappa) \ln \left(z / z_{0}\right)$. The relationship between the friction velocity $u^{*}$ and the free stream velocity $U_{\infty}$ is found to obey an affine function for the entire range of investigated velocities: $u^{*}=0.143 U_{\infty}-0.347$. Inset: Focus height as a function of the free stream velocity $U_{\infty}$.

$S_{c}^{*}$, the velocity profiles do not converge any more at a unique point. We can however still define a pseudo-focus point as the intersection point of two successive wind profiles. This pseudo-focus point is found to increase with increasing Shields number greater than $S_{c}^{*}$, suggesting that the transport layer thickens with increasing wind strength in the collisional regime (see the inset of Fig. 2).

From PIV measurements, we extracted particle velocity profiles (see Fig. 3). The horizontal particle velocity increases with increasing height for all wind strengths. In the saltation regime, we confirm that the particle velocity within the saltation layer (i.e. $z<z_{f}=40 \mathrm{~d}$ ) is almost insensitive to an increase of the wind strength, as shown by Ho et al. [7]. In contrast, in the collisional regime, the particle velocity increases dramatically with increasing wind strength. This contrasting behavior is also evidenced through the slip velocity (i.e., the velocity extrapolated at the bed [16]) which is invariant with the friction velocity in the saltation regime but increases linearly with it in the collisional regime $\left(u_{\text {slip }} \approx 0.85 u^{*}\right.$; see the inset of Fig. 3).

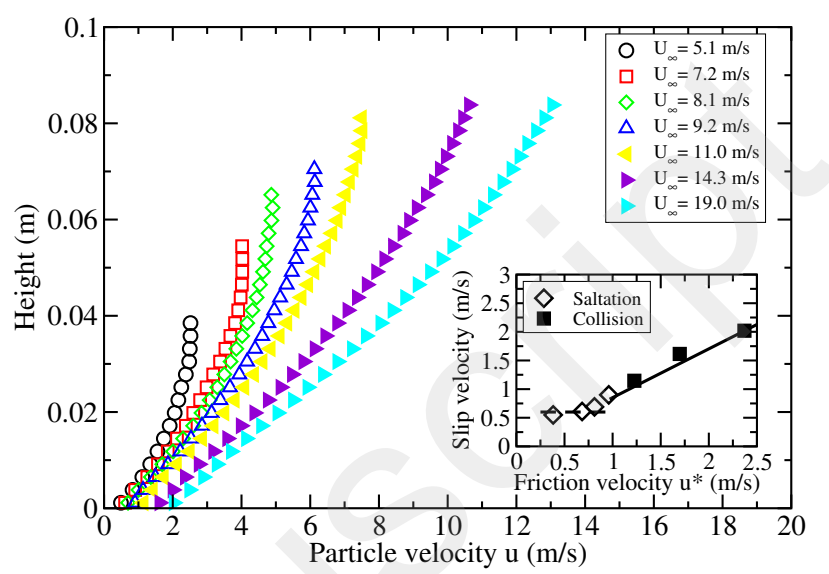

Figure 3: Mean horizontal particle velocity versus height for various flow velocity. Inset: Particle slip velocity versus the friction velocity $u^{*}$.

The last feature drawn from the experiments concerns the mass flux density profile assessed via a particle counter probe [10]. For all wind strengths investigated so far, the mass flux density was found to decrease with height at an exponential rate $q(z)=q_{0} \exp \left(-z / l_{q}\right)$, where $q_{0}$ is the flux density extrapolated at the bed surface and $l_{q}$ the characteristic decay length (see Fig. 4.a).

Interestingly, the characteristic decay length $l_{q}$ exhibits a contrasting behavior at low and large Shields number. The characteristic decay length $l_{q}$ is invariant with the Shields number in the saltation regime (i.e., $l_{q}^{\text {salt }} \approx 95 d$ ) as shown by Ho et al. [7]. However, this invariance of the decay length breaks down at large wind strengths: the latter increases with increasing Shields number in the collisional regime (Fig. 4.b). The increase of $l_{q}$ is found to follow a linear trend with the Shields number: $l_{q}-l_{q}^{\text {salt }} \approx 108 d\left(S^{*}-S_{c}^{*}\right)$. This increase indicates that the height of the transport layer increases with increasing Shields number and provides a natural explanation for the disappearance of the focus point for the air velocity profiles at high Shields number.

In contrast with $l_{q}$, the variation of the flux density $q_{0}$ with the Shields number does not show any transition between low and large Shields number (see Fig. 4.c). It follows a linear variation with the Shields number both at low and high wind strengths with the same rate: $q_{0} \approx$ $0.18 \rho_{p} \sqrt{g d}\left(S^{*}-S_{s}^{*}\right)$.

Discrete numerical simulations - We completed our experimental investigation by the development of twophase numerical simulations based on a discrete element method for particle dynamics coupled to a continuum 

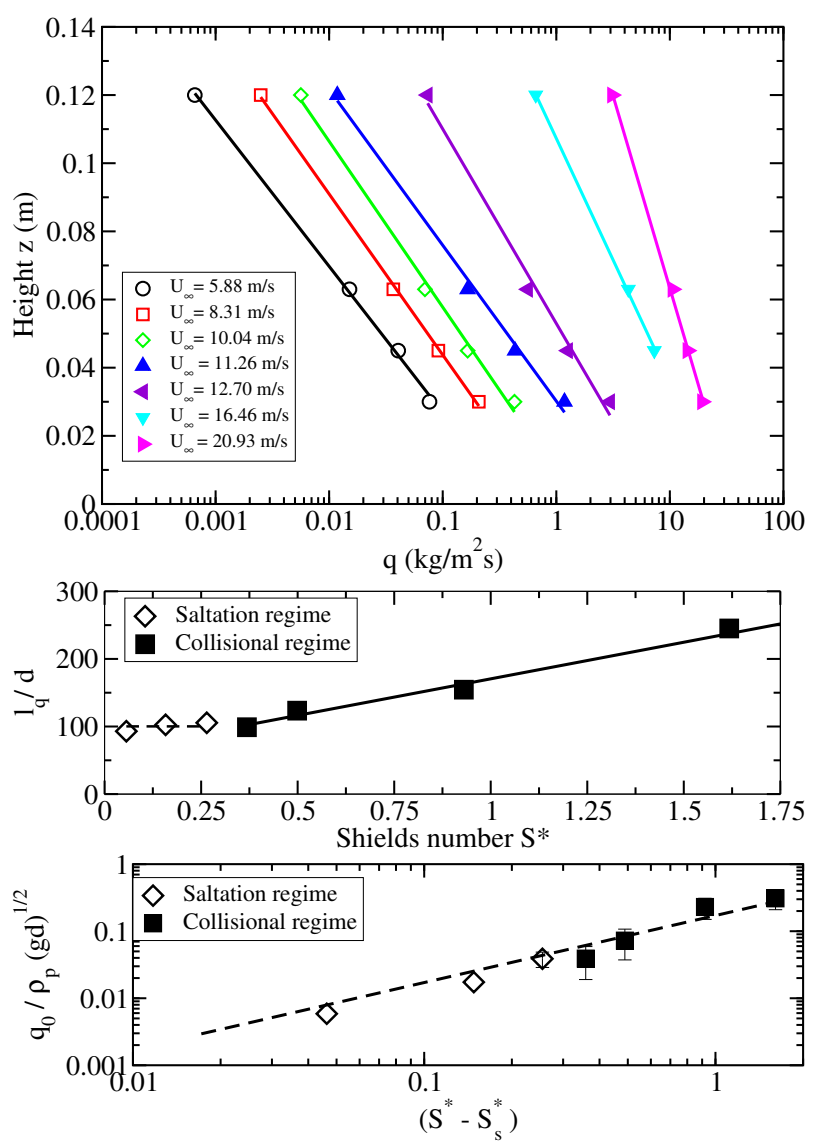

Figure 4: (a) Mass flux density profile $q(z)$ for different wind strengths. (b) Characteristic decay length $l_{q}$ versus the Shields number. (c) Flux density $q_{0}$ versus the Shields number.

Reynolds averaged description of hydrodynamics. We used the same approach as that developed by Duran et al. [15] (see further details in the Supplementary material).

The simulated system is two-dimensional (i.e. the center of mass of the particles is confined with a vertical plane). We employed periodic boundary conditions in the stream-wise direction and considered spherical particles with similar properties as those in the experiments (i.e., $d=200 \mu \mathrm{m}$ and $\rho_{p}=2650 \mathrm{~kg} / \mathrm{m}^{3}$ ). We used typical values for the normal restitution and friction coefficient, $e_{n}=0.9$ and $\mu=0.5$, respectively (See the Supplementary for further details). For these parameters, the density ratio is $\sigma=2208$ and the particle Reynolds number $R=0.5$.

The outcomes of the simulations confirms the existence of a transition between two different transport regimes at low and high Shields number. We reported on Fig. 5 the mass flow rate as a function of the Shields number obtained from the simulations which supports the change of transport regime. The numerical data indicates that the mass flow rate varies linearly with the Shields number at low wind strength up to a critical Shields number

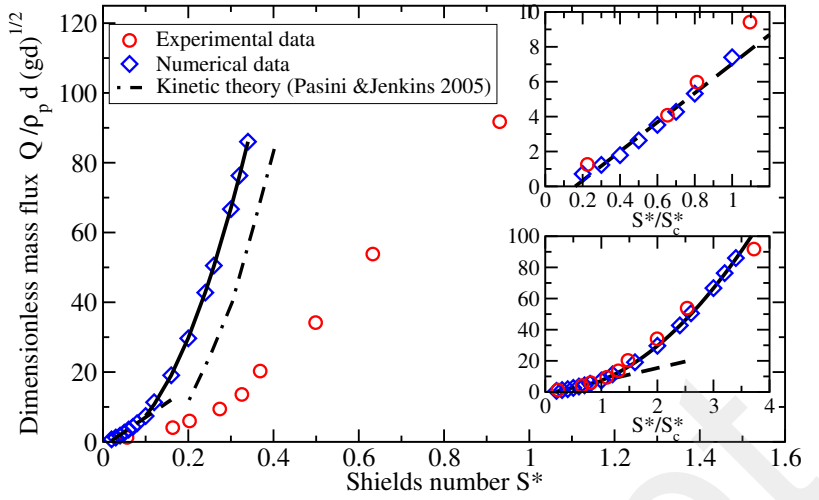

Figure 5: Mass flow rate obtained from the discrete simulations: A linear trend with the Shields number is observed up a critical $S_{c}^{*} \approx 0.1$ and a quadratic

behavior above. The saltation threshold $S_{s}^{*}$ obtained from the simulations is 0.01. Experiments and prediction from the aeolian transport model of Pasini et al. [17] based on kinetic theory are displayed as well. Insets: Experimental and numerical data re-plotted as a function the reduced Shields number $S^{*} / S_{c}^{*}$. In the upper Inset, we show a close-up view of the saltation regime.

$S_{c}^{*}=0.1$. Above this critical value, the mass flow rate clearly deviates from a linear trend and its variation is compatible with a quadratic increase with the Shields number $\left(Q \propto S^{* 2}\right)$. We thus obtain the same type of transition as that observed in the experiments. However, we can note that the agreement between simulations and experiments is not quantitative. The simulations greatly overestimate the mass flow rate and predict a transition towards the collisional regime at a lower critical Shields number in comparison with that found in the experiments $\left(S_{c}^{* \operatorname{simu}}=0.1\right.$ against $\left.S_{c}^{* \exp }=0.3\right)$. However, it is important to note that the transition occurs at the same critical mass flow rate $Q_{c} \approx 10 \rho_{p} d \sqrt{g d}$. We will come back to that point and the possible causes of the discrepancy later on.

Additionally, the mass flux density profiles obtained from the simulations exhibit the same trends as those seen in the experiments (see Fig. 6). The latter decreases at an exponential rate as a function of the distance from the bed surface. The mass flux density $q_{0}$ extrapolated at the bed surface (see the Supplementary material for the precise definition of the bed surface) increases linearly with the Shields number at low and high wind strength but the characteristic decay length indicates a change of behavior at a critical Shields number $S_{c}^{*}=0.1$ : Below this value, $l_{q}$ is almost constant and of order of $50 d$, and above it, $l_{q}$ increases linearly with the Shields number. These features are in agreement with the experiments.

Fig. 7 displays the particle velocity profiles for increasing Shields number obtained from the simulations. Interestingly, the variation of the depth-averaged particle velocity (see the Supplementary material for its compu- 

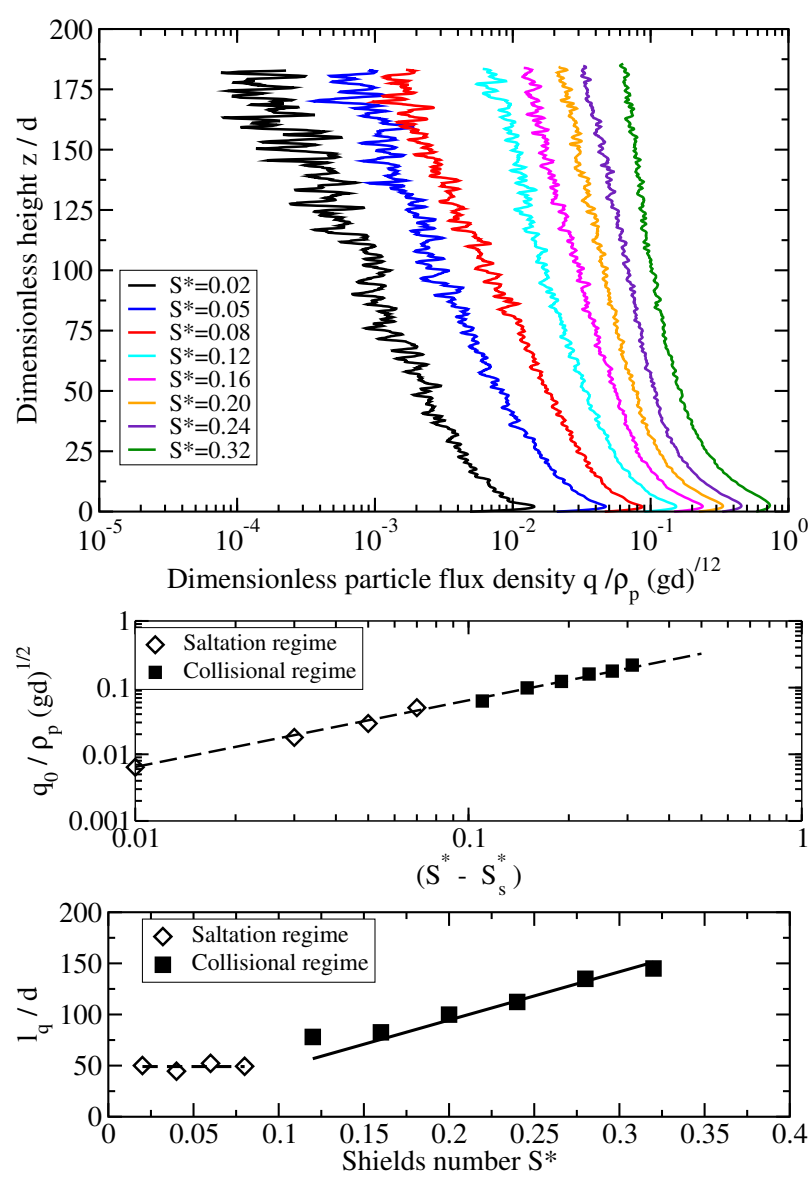

Figure 6: Simulation outcomes. (a) Mass flux density profiles for various Shields number; (b) Mass flux density $q_{0}$ at the bed versus the Shields number: $q_{0} \approx 0.62\left(S^{*}-S_{s}^{*}\right) ;(\mathrm{c})$ Characteristic decay length $l_{q}$ versus the Shields number: In the saltation regime $l_{q} / d \approx 50$ and in the collisional regime $l_{q} / d \approx 425 S^{*}$.

tation) with wind strength also highlights a transition at critical Shields number $S_{c}^{*}=0.1$ which corresponds to critical friction velocity $u^{*} \approx 4 u_{s}^{*}\left(u_{s}^{*} \approx 0.2 \mathrm{~m} / \mathrm{s}\right)$. Below it, the depth-averaged particle velocity is invariant with the wind strength as expected for saltation transport but above it, the latter increases with increasing friction velocity (see the inset of Fig. 7. The increase is linear with the friction velocity. These features corroborate the experimental results (see Fig. 3).

The last important point is to check whether the transition observed at high Shields number can be associated to a change of transport regime from saltation to collisional regime. Our hypothesis is that at large Shields number the saltation regime breaks down because midair collisions become predominant within the transport layer. To check the validity of this hypothesis, we determined the mean free path of the particles via the twophase numerical simulations (see Supplementary material). In the saltation regime, the mean free path is expected to be twice the maximum elevation height of the

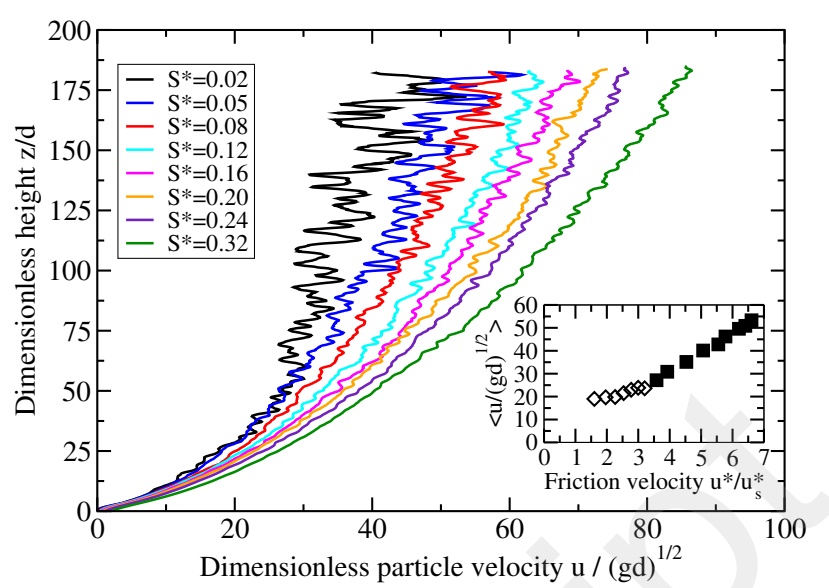

Figure 7: Particle velocity profiles obtained from the numerical simulations. Inset: Depth-averaged particle velocity versus the friction velocity.

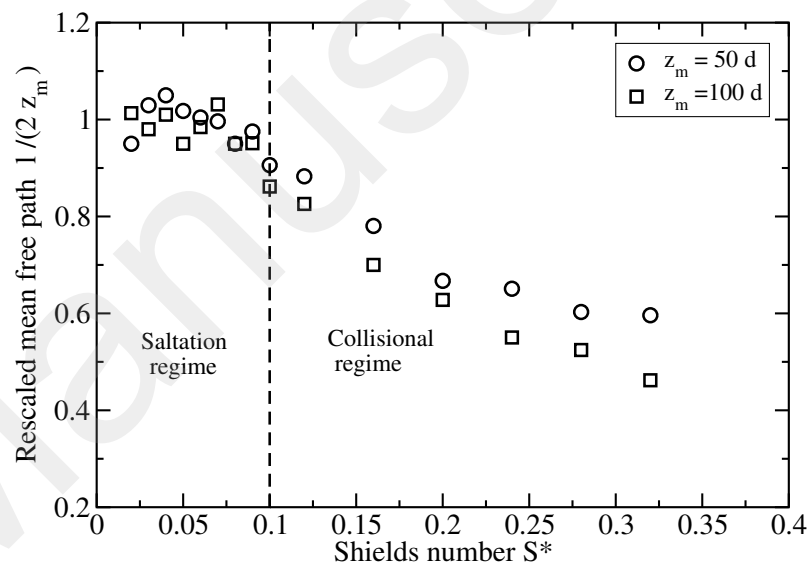

Figure 8: Rescaled mean free path $l /\left(2 z_{m}\right)$ of the saltating particles versus the Shields number. The mean free path depends on the maximum elevation height $z_{m}$ of the particle trajectory (see Supplementary material). We computed it for two different elevations $z_{m}=50 d$ and $100 d$.

particle trajectory whereas it is expected to be smaller in the collisional regime [18]. This is precisely what we found (see Fig. 8). It is also important to stress that the collisional layer remains confined close to the bed and is topped by a ballistic region free of mid-air collisions.

Conclusion- We have seen that the transition in experiments and simulations occurs at the same critical flow rate $Q_{c}$ but at different critical Shields number $S_{c}^{*}$. This call for severals concluding remarks. (i) First, the fact that $Q_{c}$ is similar in experiments and simulations gives credit to the hypothesis that the transition is driven by mid-air collisions. The mass flow rate is, in the saltation regime, directly proportional to the depth-averaged particle volume (since the particle velocity and transport layer thickness remain constant), and is thus expected to control the onset of particle collisions within the trans- 
port layer. (ii) Second, the underestimation of $S_{c}$ in the simulations seems to originate from a general overestimation of the simulated mass flow rate. As a result, the transition predicted by the simulations is expected to occur at a lower critical Shields number, which is indeed observed. Interestingly, if we re-plot the mass flow rate curves rescaling the Shields number by the critical value $S_{c}$, we get a nice collapse between the experimental data and the simulations (see the inset in Fig. 5). One plausible explanation for the overestimation of the simulated mass flow rate may be found in the simplified descrip- tion of the granular phase (e.g., spherical shape, simple visco-elastic model for particle-particle interactions). In particular, the particle shape can significantly affect the mass flow rate as mentioned in [19, 20]. Additionally, the turbulent flow model employed in the simulations which is based on classical mixing turbulent length could be eventually improved to account for the possible damping of the turbulence by the saltating particles.

This work has been supported by the French National Agency for Research (Grant No. ANR-17-CE01-0014).
[1] R. A. Bagnold. The physics of blown sand and desert dunes. Methuen, New York, 1941.

[2] O. Duran, P. Claudin, and A. Andreotti. Aoelian Research, 3:243-270, 2011.

[3] J.F Kok, E.J.R Parteli, T.I Michael, and D. B. Karam. Report on progress in Pysics, 75:106901, 2012.

[4] A. Valance, K.R. Rasmussen, A. Ould El Moctar, and P. Dupont. Comptes Rendus Physique, 16:105-117, 2015.

[5] M. V. Carneiro, N.A.M. Araujo, T. Pähtz, and H. J. Herrmann. Phys. Rev. Lett., 111:058001, 2013.

[6] O. Duran and T. Pähtz. Physical Review Fluids, 2:074303, 2017.

[7] T. D. Ho, A. Valance, P. Dupont, and A. Ould El Moctar. Physical Review Letters, 106:094501, Mar 2011.

[8] M. Creyssels, P. Dupont, A. Ould El Moctar, A. Valance, I. Cantat, J.T. Jenkins, J.M. Pasini, and K.R. Rasmussen. Journal of Fluid Mechanics, 625:47-74, 2009.

[9] Takeshi Sato, Tadashi Kimura, Taminoe Ishimaru, and Toshisuke Maruyama. Annals of Glaciology, 18:149-154, 1993.

[10] K. Nishimura, and M. Nemoto. Antarctica, Phil. Trans. R. Soc. A, 363:1647-1662, 2005.

[11] A correcting factor is used to account for the effect of the side-wall (see Supplementary material for further details, which includes $[12,13])$.

[12] T. D. Ho. Phd Thesis, University of Rennes, France, 2012.

[13] H. Selmani, A. Valance, A. Ould El Moctar, P. Dupont, and R. Zegadi Geophysical Research Letters, 45:18381844 (2018).

[14] P. D. Julien. Erosion and sedimentation, Cambridge University Press, 1995.

[15] O. Duran, B. Andreotti, and P. Claudin. Phys. Fluids, 24:103306, 2012.

[16] The slip velocity is determined via a linear extrapolation of the velocity profile down to the bed surface. The profile is measured with a good accuracy down to a height $z=$ $2 \mathrm{~mm}$ above the bed surface.

[17] J.M. Pasini and J.T. Jenkins. Phil.Trans.R. Soc. A, 363:1625-1646, 2005.

[18] Diego Berzi, James T. Jenkins, and Alexandre Valance. Journal of Fluid Mechanics, 786:190-209, 2016.

[19] T. Pähtz. and O. Duran. Phys. Rev. Fluids, 10:104302, 2018.

[20] Y. Chen, and J. Zhang and N. Huang and B. Xu Journal of Geophysical Research: Atmospheres, 124, 7226-235, 2019. 